\title{
Critical fail concepts in surgery: highlighting the "must-know" concepts
}

\section{Arman Abdalkhani, MD \\ Geoffrey Blair, MD}

Accepted July 28, 2020

\section{Correspondence to:}

A. Abdalkhani

Department of Surgery

Faculty of Medicine

The University of British Columbia

11th Floor - 2775 Laurel St

Vancouver BC V5Z $1 \mathrm{M} 9$

dr.a@ubc.ca

DOI: $10.1503 /$ cjs.020019

\section{SUMmaRY}

As the vast majority of medical students do not pursue further postgraduate surgical education, we suggest that failing to achieve certain learning objectives (LOs) has greater potential consequences than others. We developed a tiered LO architecture that classifies a high-priority subset of "critical fail" LOs within the current surgical learning objectives for medical students. Followup of student performance on written examinations revealed an improvement in applying critical fail concepts without sacrificing performance on other LOs. Here we describe how we modified our declared curriculum to incorporate these changes.
A t medical training institutions across Canada, $90 \%$ of graduating medical students do not enter postgraduate surgical specialty traineeships. ${ }^{1}$ As the vast majority of students do not pursue further postgraduate surgical education, we suggest that failing to achieve certain learning objectives (LOs) has greater potential consequences than others.

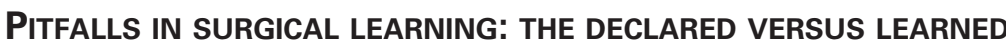 CURRICULUM}

The scope of surgery and its subspecialties has exploded in recent years, ${ }^{2}$ and surgical educators are faced with prioritizing which information becomes part of the surgical curriculum in medical education. Balancing the new guidelines for care while still adhering to the principles of surgical training outlined by William Halsted ${ }^{3}$ many decades ago has resulted in a stepwise progression toward educational curricula with defined LOs. Harden ${ }^{4}$ defined a teaching approach as a process in which taught objectives (i.e., what is taught), the teaching method and timing (i.e., how and when it is taught), and finally the assessment (i.e., the measures used to determine whether the student has achieved the expected learning outcomes) is integrated.

Standardization of clinical medical education is difficult given the variety of teaching styles, subspecialty availability/teaching capacity, and patient populations encountered by students. Therefore, the curriculum framework proposed by $\operatorname{Harden}^{3}$ helps educators address the delivery of LOs. In this framework, 3 different kinds of curricula should be distinguished from one another:

- declared curriculum - the curriculum intended by the faculty

- taught curriculum - the content taught by the educator

- learned curriculum - the LOs achieved by the students

Distinguishing among these types of curricula can reveal if there are gaps on how and when intended knowledge is transmitted to the student. In a recent article by Sterz and colleagues, ${ }^{5}$ less than $60 \%$ of the 83 declared curriculum LOs were actually addressed in the 32 surgical lectures delivered at their institution. To our knowledge, this is the only publication that addresses the gaps between declared and taught curriculum, and we sought to elucidate any 
gaps between declared and learned curriculum in the undergraduate surgical education program at our institution.

Although the declared curriculum is addressed during our third-year clerkship surgical lecture/seminar series, which every student attends, clinical exposure and personal reading, which are also part of the taught and learned curricula, vary from student to student. We were alerted to what appeared to be a significant gap between the declared curriculum and learned curriculum at our institution after review of our written examination results.

\section{UNDERSTANDING THE CURRICULUM GAP: AUDITING EDUCATIONAL PERFORMANCE}

Multiple strategies for assessing the gap between the declared and taught curricula could be pursued. At our institution, we distribute the declared curriculum in the form of LOs organized according to surgical specialties at the onset of the 8 -week third-year surgical rotation. Formative and summative assessment of student knowledge are available to our medical educators and include clinical evaluations, online modules, and traditional written examinations. Therefore, for the purposes of analyzing the gap between the declared and learned curriculum, we reviewed the number of correct responses from 144 third-year examinations at our institution. The written exam is a multiple-choice question exam (MCQE) consisting of 120 questions and is administered at the conclusion of the 8-week surgical rotation.

Upon reviewing the examinations, it became apparent that a percentage of our students had not learned some key clinical concepts in surgery - concepts that, if not understood, could easily result in future clinical errors with lifeor limb-threatening consequences. We identified a subset of the MCQE questions that addressed these clinical scenarios that carried high clinical significance. As a result, we identified a high-priority list of LOs that would benefit the student, which we refer to as "critical fail learning objectives" (CFLOs). The updated LOs would then be distributed to the next group of students to underscore their importance. We then analyzed the subsequent set of MCQE questions after the release of the modified LOs to see if there was an improvement in performance in those questions, which addressed the CFLOs.

\section{Proposed fRAmework: the deClared CURRICULUM AND CFLOs}

Our third-year surgical clerkship's declared curriculum has a total of 156 LOs. To provide structure to these LOs and not overwhelm the students with their sheer number, they are grouped according to surgical specialty or topic (Table 1). A new designation was made within each list of LOs of those considered to be CFLOs. The basic design of the declared curriculum and the distribution of learning objectives are presented in Figure 1 and Table 1.
Table 1. Distribution of learning objectives and critical fail learning objectives, by subdivision*

\begin{tabular}{|lcc|}
\hline Specialty & $\begin{array}{c}\text { No. of learning } \\
\text { objectives }\end{array}$ & $\begin{array}{c}\text { No. of critical fall } \\
\text { learning objectives }\end{array}$ \\
\hline Thoracic surgery & 14 & 6 \\
\hline Urology & 10 & 5 \\
\hline Plastic surgery & 18 & 3 \\
\hline Otolaryngology & 12 & 4 \\
\hline Endocrine surgery & 5 & 4 \\
\hline Neurosurgery & 20 & 4 \\
\hline General surgery & 42 & 7 \\
\hline GI/breast/hepatic surgery & 9 & 5 \\
\hline Pediatric surgery & 8 & 4 \\
\hline Vascular surgery & 11 & 1 \\
\hline Trauma surgery & 7 & 44 \\
\hline Pre- and postoperative care & 156 & \\
\hline Total & & \\
\hline CFLO = critical fail learning objective; GI = gastrointestinal; LO = learning objective. \\
$*$ The previous 156 LOs now have 44 CFLOs identified after faculty review and present a \\
smaller subset of LOs that the student can make a high priority.
\end{tabular}

We had solicited direct input from each subspecialty/ divisional/departmental head in order to formulate each CFLO subset of the declared curriculum. The list of surgical learning objectives and the CFLO subsets are passed on as an electronic handout to the medical students at the beginning of their surgical rotation. Box 1 shows an example for vascular surgery.

\section{Discussion}

We believe that prioritizing the declared curriculum helps guide the student in understanding the CFLOs without diluting or detracting from the other LOs. A literature search revealed no previous studies that directly addressed prioritizing surgical LOs so that students can focus on the most important ones in the curriculum. To help clarify if this guidance was helpful, we also adjusted the taught curriculum and directly addressed these LOs by adding a mid-rotation self-test that focused heavily on CFLOs. The self-test consisted of an MCQE with an answer key that had explanations of the correct answers.

We compared the performance of the students with the updated LO list and exposure to the mid-rotation self-test to that of the previous student group by auditing the endof-rotation MCQE test. The results showed that students who were given the modified CFLO handout and the selftest scored considerably higher on questions that addressed the CFLOs without adversely affecting performance on questions that addressed the other LOs.

We realize that by highlighting CFLOs, students may consider the other LOs to be extraneous. To ensure that students continue to study the standard LOs, they are reminded in multiple academic full-day sessions that more than $70 \%$ of the end-of-rotation MCQE questions focus on the traditional LOs. 


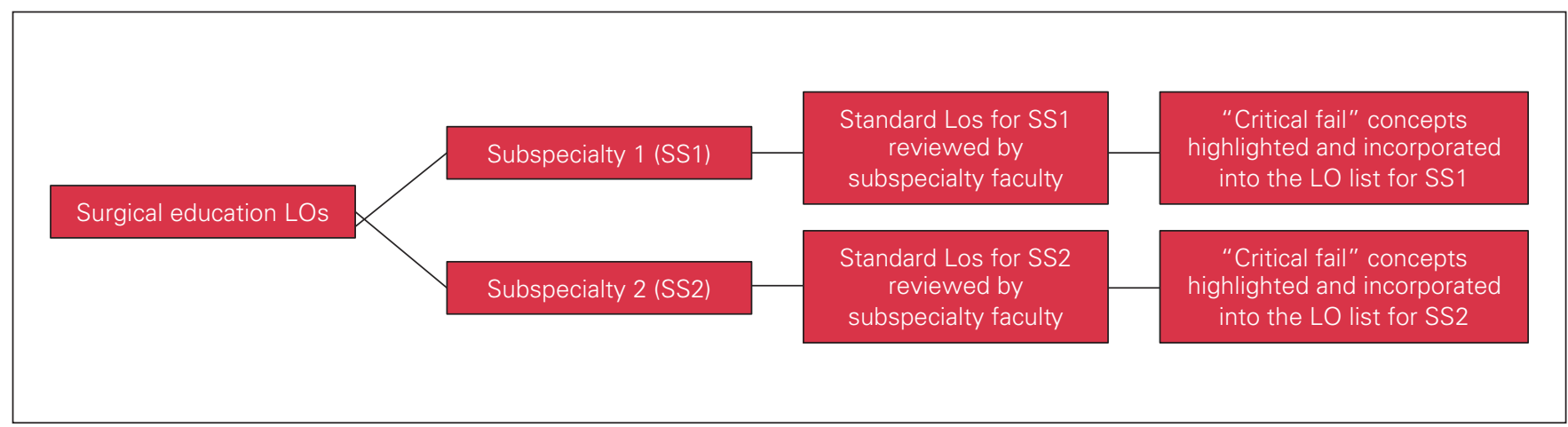

Fig. 1. Approach for the design of prioritized learning objectives (LOs) at the authors' institution. The large number of LOs are subdivided into smaller groups based on subspecialty and faculty experts review the current LOs to create a subset of "critical fail" LOs.

\section{Box 1: Vascular surgery third year learning objectives}

1. Discuss the management of a 65-year-old male who presents with abdominal ultrasound evidence of a $6.0 \mathrm{~cm}$ abdominal aortic aneurysm.

2. Perform a focused surgical history and physical examination and outline urgent procedures and diagnostic tests required for a patient presenting with signs and symptoms suggestive of a ruptured aortic aneurysm.

3. Discuss the investigations, treatment and general management of an 80-year-old female who presents with a carotid bruit and compare both medical and surgical therapy for extracranial carotid occlusive disease in patients with symptomativ versus asymptomatic stenosis

4. Outline the approach to a 78-year-old man with an acutely painful, pale, pulseless leg giving reference to the natural history of acute arterial occlusion and its irreversible effects.

5. Describe the diagnostic approach and management scheme with treatment options for a patient presenting with intermittent claudication.

6. Explain the significance of Virchow's triad and its importance in chronic venous insufficieincy.

7. Characterize deep venous thrombosis in the context of the postoperative patient and outline the approach to investigation and treatment.

8. Describe the pathophysiology of pulmonary embolus and its usual clinical manifestations in the postoperative patient.

Critical fail concepts

- Painful, pulseless limb

- Aortic aneurysm - both enlarging and rupture scenarios

- Cerebral ischemic threats (carotid stenosis)

- Deep vein thrombosis/pulmonary embolism

\section{Conclusion}

Early data from our cohort of 144 student exams that were reviewed show a relative increase in the mean scores of MCQE questions that related to CFLOs. Prior to the release of the modified learning sheets and practice questions, a mean score of $71 \%$ was achieved across 23 pertinent examination questions; the mean score after introducing these tools increased to $82 \%$. Scores on non-CFLO questions did not change appreciably across the cohort group.

Our hope is to eventually have students pass if they 1) achieve both a passing overall surgical grade based on MCQE score and clinical rotation assessments, and
2) never failed to recognize the correct answer to a straightforward CFLO clinical scenario. This desired dual requirement for passing the surgery rotation will be difficult to achieve, but we believe the new CFLOs are a starting point to address "critical fail" surgical concepts and improve students' understanding of them to a good and measurable effect. We hope to further refine the delivery and evaluation of this methodology to continue narrowing the gap between the declared and learned curricula in undergraduate surgical education programs.

Affiliations: From the Department of Otolarynogology, University of British Columbia, Vancouver, BC (Abdalkhani); and the Department of Surgery, University of British Columbia, Vancouver, BC (Blair).

\section{Competing interests: None declared.}

Contributors: Both authors contributed substantially to the conception, writing and revision of this article and approved the final version for publication.

Content licence: This is an Open Access article distributed in accordance with the terms of the Creative Commons Attribution (CC BYNC-ND 4.0) licence, which permits use, distribution and reproduction in any medium, provided that the original publication is properly cited, the use is noncommercial (i.e., research or educational use), and no modifications or adaptations are made. See: https://creativecommons. org/licenses/by-nc-nd/4.0/

\section{References}

1. CARMS Match Data. Ottawa: CaRMS; year Available: https://www. carms.ca/data-reports/r1-data-reports/r-1-match-interactive-data/ (accessed 2019 Mar. 15).

2. Webber EM, McAlister VC, Gorman L, et al. The past and future of the generalist general surgeon. Can 7 Surg 2014;57:6-7.

3. Halsted WS. The training of the surgeon. Bull fohns Hopkins Hosp 1904;15:267-75.

4. Harden RM. AMEE Guide No. 21: Curriculum mapping: a tool for transparent and authentic teaching and learning. Med Teach 2001;23:123-37.

5. Sterz J, Hoefer S, Janko M, et al. Do they teach what they need to? An analysis of the impact of curriculum mapping on the learning objectives taught in a lecture series in surgery. Med Teach 2019; 41:417-21. 